\title{
The pharmacological profile of a novel highly potent
}

bisphosphonate, OX14, with reduced bone affinity, is as effective as zoledronic acid at inhibiting bone resorption in normal growing mice and rats and in the treatment of myeloma bone disease in JJN3-NOD/SCID-Y mice

\author{
M A Lawson 1,2, F H Ebetino3 ${ }^{3}$ A Mazur ${ }^{4}$, A D Chantry ${ }^{1,2}$, J Paton-Hough1,2, H R Evans ${ }^{1,2,}$, D Lath 1,2, M K
}

Tsoumpra1,2, M W Lundy ${ }^{5}$, R L M Dobson ${ }^{6}$, M Quijano $^{6}$, A A Kwaasi ${ }^{7}$, J E Dunford7, X Duan , J T

Triffitt $^{7}, \mathrm{G}$ Jeans $^{6}$ and R G G Russell ${ }^{1,2,7}$

The

University

Of

'Department of Oncology and Metabolism \& ${ }^{2}$ Mellanby Centre for Bone Research, Medical School,

Sheffield.
University of Sheffield, UK, ${ }^{3}$ University of Rochester, Rochester, NY, USA, ${ }^{4}$ TWI Chem LLC, Mason, Ohio,
USA, ${ }^{5}$ Indiana University, Indianapolis, IN, USA, ${ }^{6}$ Procter \& Gamble, Mason, Ohio, USA. ${ }^{7}$ The Botnar

Research Centre, Nuffield Orthopaedic Centre, University of Oxford, UK.

\section{Bloodwise}

Beating blood cancer since 1960
Introduction

Bisphosphonates are widely used in the treatment of clinical disorders characterised by increased bone resorption, including osteoporosis, Paget's disease and the skeletal complications of malignancy. The antiresorptive potency of the nitrogen-containing bisphosphonates on bone in vivo is now recognised to depend upon two key properties, namely mineral binding affinity and inhibitory activity on farnesyl pyrophosphate synthase (FPPS), and these properties vary independently of each other in individual bisphosphonates. The better understanding of structure activity relationships among the bisphosphonates has enabled us to design a series of novel bisphosphonates with a range of mineral binding properties and antiresorptive potencies. Among these is a highly potent bisphosphonate with reduced bone affinity, namely 1-fluoro-2-(imidazo-[1,2 alpha]pyridin-3-yl)ethyl-bisphosphonate, also known as OX14 (Fig. 1). The aim of this work was to characterise OX14 pharmacologically in relation to other bisphosphonates currently used clinically.

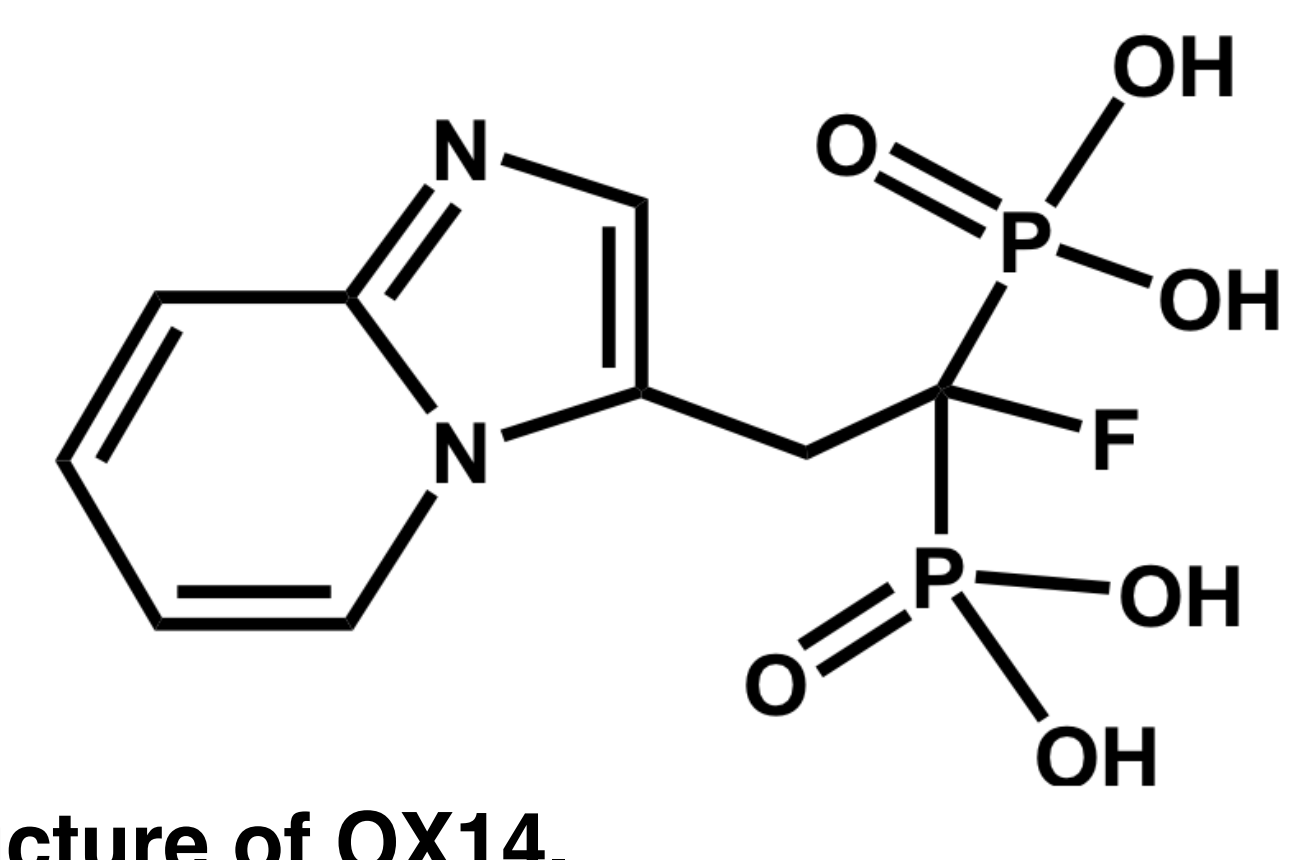

Figure 1. The chemical structure of $0 \times 14$

\section{Methods}

\section{In vitro evaluation of OX14 compared to other bisphosphonates}

The retention and binding of OX14 was compared to alendronate (ALN), zoledronic acid (ZOL), ibandronate (IBN) and risedronate (RIS) on a hydroxyapatite (HAP) column. ${ }^{1}$ The potential anti-resorptive potency of OX14 was compared to ALN, ZOL, IBA and RIS in a FPPS inhibition assay. ${ }^{2}$

In vivo evaluation of OX14 compared to other bisphosphonates

In a growing rat model ${ }^{3}$ and in a growing mouse model the effects of OX14 on bone binding affinity and bone resorption were compared to other bisphosphonates. The therapeutic effects of OX14 were assessed in the JJN3-NSG murine model of myeloma and compared to ZOL. Groups of female NSG mice $(n=8)$ were injected iv with $1 \times 10^{6}$ JJN3 cells and treated with Vehicle, ZOL or OX14 (125 $\mu \mathrm{g} / \mathrm{kg} \mathrm{sc} \mathrm{2x/wk).} \mathrm{Micro-CT}$ analysis was used to measure trabecular bone volume and number of cortical bone lesions. The numbers of osteoclasts on tibial cortico-endosteal surfaces were assessed using standard histomorphometric methods. Tumour burden was assessed in bone marrow flushes of the left femora at the end stage of disease by flow cytometry using an anti-human HLA-ABC-APC antibody.

\section{Results}

When compared to ALN, IBN, RIS and ZOL, OX14 was the most potent at inhibiting FPPS in vitro, and had the lowest binding affinity to HAP columns (Fig.2). When injected iv into Sprague Dawley growing rats, OX14 was excreted into the urine to a greater extent than other bisphosphonates, indicating lower skeletal retention, and it resulted in increased BMD compared to RIS (Fig. 2).
Binding to HAP

ह्ञ
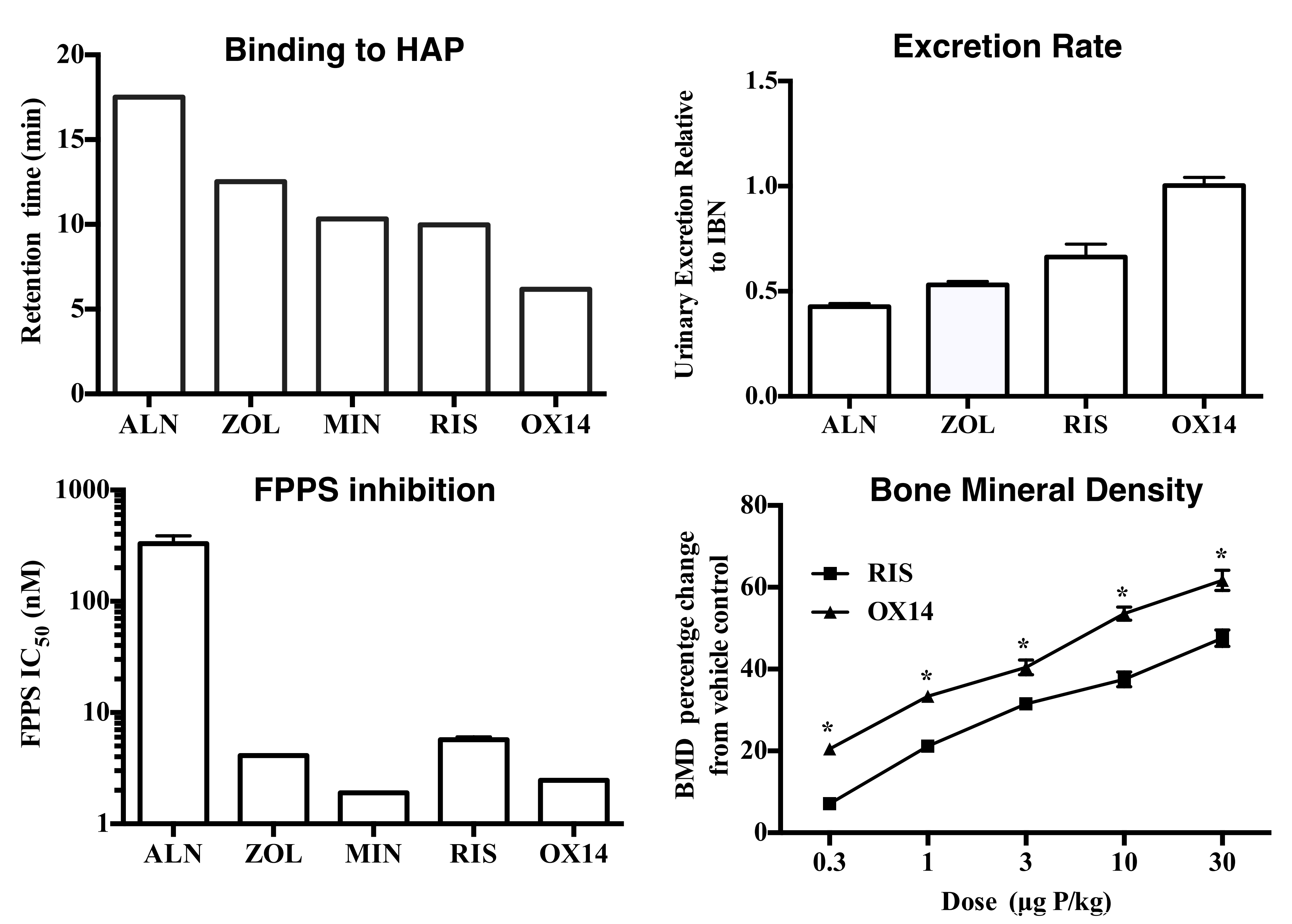

Figure 2. OX14 has a low bone binding affinity and is highly potent at inhibiting FPPS.
In studies in both rats (Fig.2) and mice (Fig. 3), OX14 showed a dose dependent inhibition of bone resorption, with an antiresorptive potency equivalent to or greater than the comparator bisphosphonates.
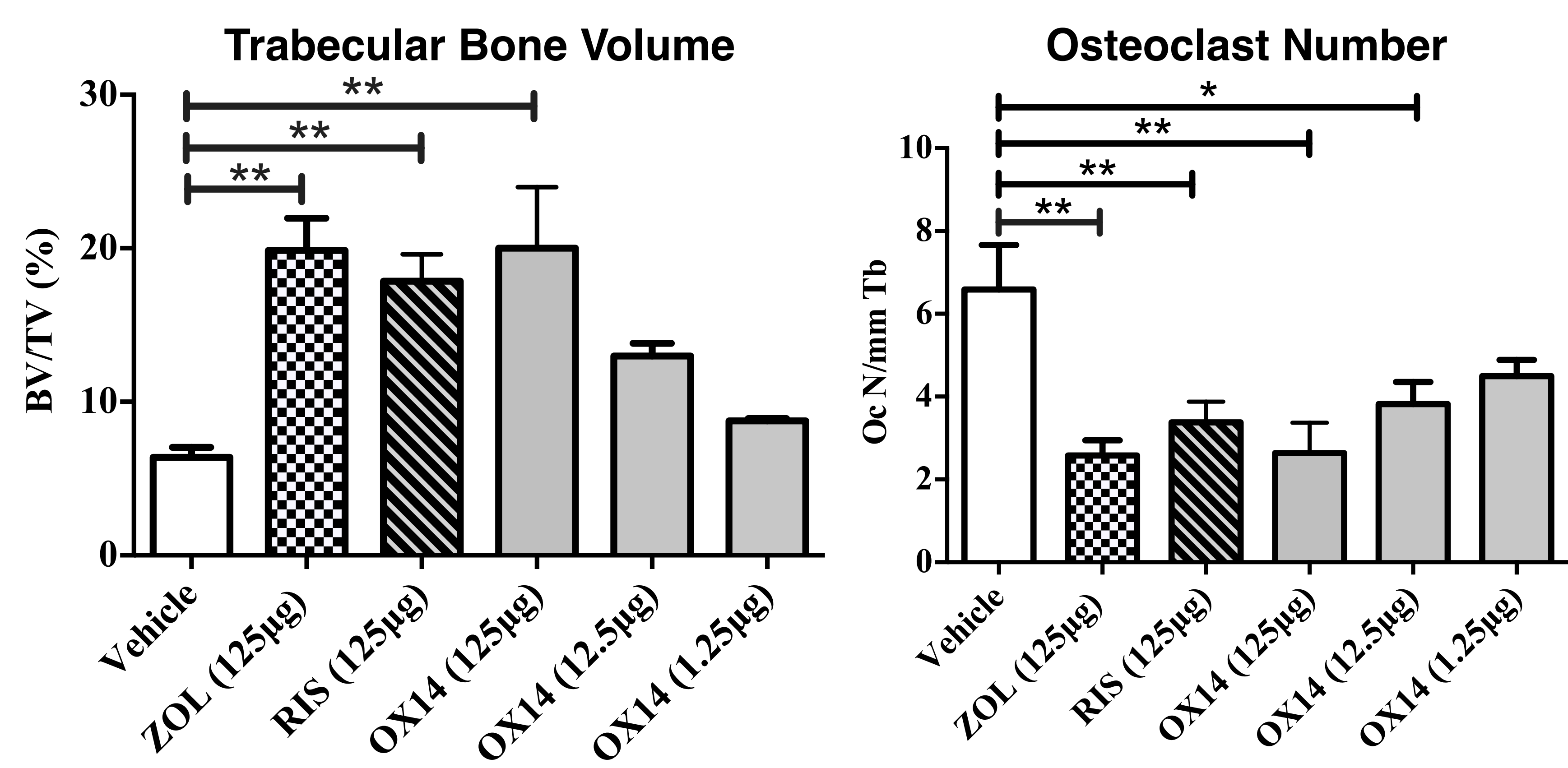

Figure 3. Evaluation of different doses of OX14 in C57BL/6J6 mice.

In the JJN3-NSG murine model of myeloma-induced bone disease, OX14 was shown to be as effective as ZOL at preventing the formation of osteolytic lesions (Fig. 4).
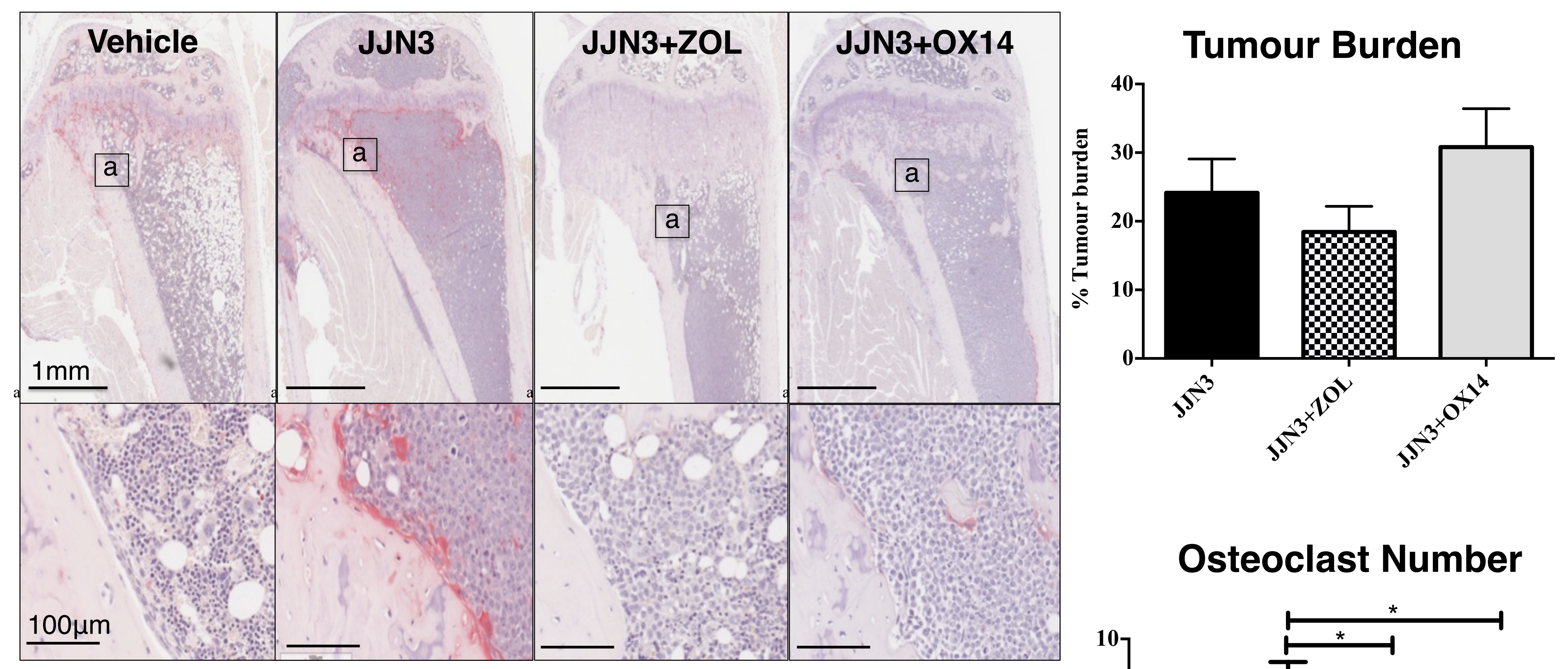

Osteoclast Number
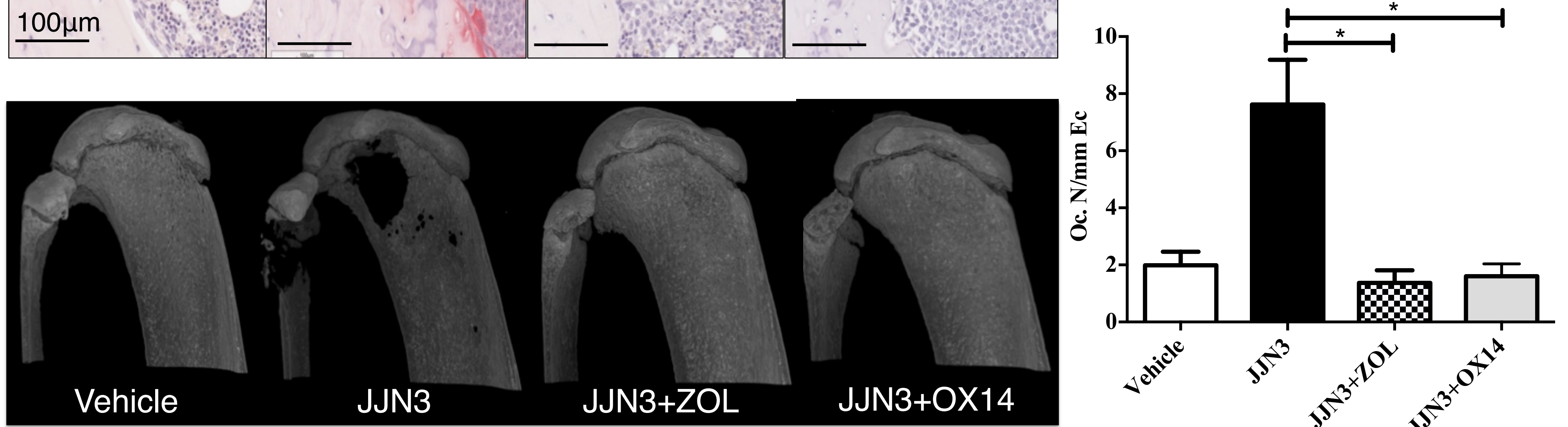

Lesion area

Trabcular Bone Volume
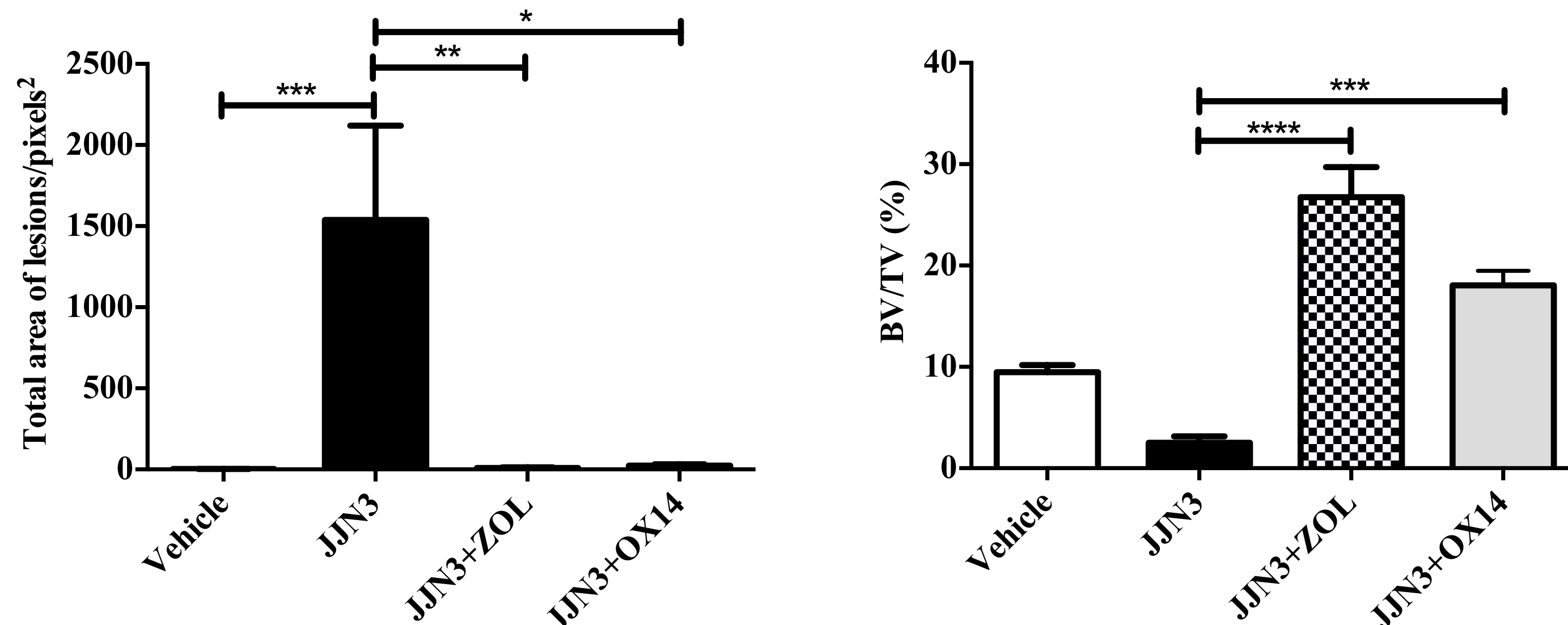

Figure 4. OX14 prevents osteolytic disease in the JJN3-NSG murine model of myeloma.

Conclusions

In summary, OX14 is a new highly potent bisphosphonate with lower bone affinity than other clinically relevant bisphosphonates. This renders OX14 an attractive candidate for clinical development for its potential skeletal or non-skeletal benefits.

\section{Acknowledgements}

We like to thank Bloodwise and Procter \& Gamble for supporting this work Lawson M.A., et al. (2010). Differences between Bisphosphonates in Binding Affinities for Hydroxyapatite. Joumal or Biomedical Materals Research Part B - Applied Blomaterials, 92 (1).149-155.

Dunford, J.E., et al., (2001). Structure-activity relationships for inhibition of farnesyl diphosphate synthase in vitro and inhibition of bone resorption in vivo by nitrogen-containing bisphosphonates. J Pharmacol Exp Ther, 296(2): 235-242.

Lundy, M., et al. (2014). Skeletal Retention and Urinary Excretion of Nitrogen-Containing Bisphosphonates Including Fluorescently-labeled Bisphosphonates in Rats. Journal of Bone and Mineral Res. 29(S1): S262. (abs 